\title{
EXPERIMENTAL ELECTRO COAGULATION UNIT FOR PRETREAMENT OF MAINS WATER FOR DTEAM GENERATION AT THERMAL POWER PLANTS
}

\author{
Xayrulla Isaxodjayev ${ }^{1}$, Nizonjon Usmonov $^{1}$, Yusufbek Abdullabekov ${ }^{1}$, Zoxid Xasanov $^{1}$ \\ ${ }^{1}$ Tashkent state technical universityengineering, Department heat power engineering, University - 2, Tashkent, 100097, Uzbekistan
}

\begin{abstract}
The article covers the state of pre-chemical treatment of natural water at Tashtpp with the use of aluminum sulphate and the creation of an experimental unit for producing coagulant, aluminum sulphate, as well as a block diagram of the proposed electrocoagulation unit for pre-treatment and clarification of water. An electrocoagulation technology for pre-treatment and clarification of water for Tashtpp steam boilers has been developed.
\end{abstract}

\section{Introduction}

Among the most important technological processes of modern thermal power plants (TPP) is a complex of processes for preparing deeply desalinated water, providing a normalized composition of impurities in the steam and feed water of boilers. For this purpose, each TPP has a system of water treatment plants (WTP). The WTP system includes: a chemical water treatment plant designed for chemical treatment of additional water that makes up for losses in the water-steam path and the heating network, as well as for cleaning condensate returned from production and installation, designed for corrective treatment of turbine condensate and feed water by introducing corrective reagents into the condensatefeed path in accordance with the accepted water-chemical regime [1].

\section{Theory and data reduction}

Usually, chemical water treatment (CHVT) consists of a pre-treatment plant for water and a subsequent chemical desalination plant. Pre-treatment consists in clarifying the source water by removing coarse and colloidal impurities from it.

For this purpose, water treatment practices use two methods:

- clarification and partial discoloration of water without pre-chemical treatment (settling, pre-and slow filtration, centrifugation or flotation);

- clarification and discoloration of water, based on the introduction of coagulants into the water (aluminum sulfate, iron (III) chloride, iron (II) sulfate, mixed aluminum-iron coagulant, etc.), which as a result of hydrolysis form poorly soluble flake-like metal hydroxides. The latter in the process of formation adsorb the suspensions and colloids contained in the water and are removed from the water by subsequent precipitation and filtration. The use of coagulants can significantly speed up the process of clarification and discoloration of water, and, consequently, reduce the size of treatment facilities and thereby reduce capital costs for their construction [2].

According to modern concepts, the process of artificial discoloration and clarification of water proceeds as follows. When a solution of coagulants is added to the water, $\mathrm{Al}_{2}\left(\mathrm{SO}_{4}\right)_{3}$ or $\mathrm{FeCl}_{3}$ during the first 30-180 seconds, the added salts are hydrolyzed to form colloidal aluminum or iron hydroxides, which have a huge active surface of the hydroxide particles.

It was found [3] that aluminum hydroxide obtained by electrochemical method has a significantly higher sorption capacity in relation to a number of water pollutants (humus, iron, silicon, etc.) than aluminum hydroxide formed as a result of hydrolysis of its salts. The latter can be explained by the special conditions for the formation of coagulation structures in electrolysis, as well as by the much smaller influence of strong acid anions, whose concentration in this case is much lower than when chemical reagents are introduced into water.

Due to the high sorption capacity of the electrochemical resulting aluminum hydroxide in the application of the electrocoagulation method is rational for pre-treatment of water in the heat power industry, semiconductor manufacturing, electric vacuum industry, etc.

The reagents used in water treatment technology are usually solid substances (aluminum sulphate, iron chloride, lime) that are fed into the water either in the form of solutions of a given concentration (wet dosing), or in the form of carefully crushed and dried powder (dry dosing).

In practice, the method of wet dosing is mainly common, in which the reagents need to be pre-dissolved.

Usually for the treatment of turbid waters the dose of coagulant is determined based on empirical data and is for 
the interval $\mathrm{Al}_{2}\left(\mathrm{SO}_{4}\right)_{3}$ of suspended matter 500-2000<smiles>C1C[As]2CCC12</smiles>

The enlargement of colloidal particles formed during the hydrolysis of coagulants occurs gradually over a long time. The appearance of visible flakes is preceded by the formation of fragile loose meshes (the so-called stage of hidden coagulation) and supermicellar structures. The latter are then torn into separate flakes, which settle under the influence of gravity.

The process of coagulation from the microscopic side is characterized by several stages. When the coagulant solution is mixed with water, no external changes are observed. After a while, opalescence appears and the water becomes cloudy from the formation of a huge number of tiny flakes up to 500 in 1 (the beginning of flake formation). These flakes are aggregated into larger ones, and the number of flakes per unit volume decreases. When it reaches $5 \div 10$ in 1 (the end of flake formation), a noticeable precipitation of flakes begins.

For intensive clarification and discoloration of water, it is usually sought to obtain light-settling large flakes with a highly developed surface. At the same time, they try to reduce the time of formation of flakes. The mechanism of flake formation is determined by the probability of colliding colloidal particles, which depends on their concentration, mobility and order of movement, as well as their coupling.

It is found that in the temperature range of $3-18^{\circ} \mathrm{C}$ when mixing, the formation of flakes takes $2-7 \%$ of the time necessary to achieve the same effect without mixing, and the flakes are obtained more durable. To prevent flake destruction, it is necessary to keep the flakes in a suspended state with little stirring.

When settling, the main mass of suspended substances is removed from the treated water.

In vertical settling tanks the suspension particles are under the action of two opposite forces: the upward flow of water and the mass of the particle. The speed in vertical settling tanks is assumed to be equal to the speed of the vertical upward flow of water in them $(0.35-0.6 \mathrm{~mm} / \mathrm{s}$ in the case of settling water treated with coagulants).

In a vertical cylindrical sump with a conical bottom, water enters the Central pipe, passes down it, and then rises up through the working part of the sump. At the exit from the Central pipe, the speed of water movement changes sharply, which in combination with the centrifugal forces that occur when the flow movement turns sharply, leads to precipitation. The accumulated sediment in the conical part of the sump is periodically discharged into the sewer (the sediment is released by squeezing it out with a column of water located above it).

Currently, vertical settling tanks are replaced by clarifiers, in which the water is clarified by a layer of suspended sediment.

Operating practice shows that the efficiency of clarification and discoloration of water when passing it through suspended sediment is about one and a half to two times greater than in conventional vertical settling tanks.
In the water treatment plant (WTP) of TashTPP, an important part is the pre-treatment of water from silt and various organic impurities. Pre-treatment consists in clarifying the source water (Boz-su channel) by removing coarse and colloidal impurities from it. Until now, water clarification has been performed by coagulation by introducing a special coagulant into the treated water (in the case of TashTPP, this is aluminum sulfuric acid $\mathrm{Al}_{2}\left(\mathrm{SO}_{4}\right)_{3}$.

We have developed an electro coagulation technology for pre-treatment and clarification of water for TashTPP steam boilers.

The essence of the electro coagulation method consists in the anodic dissolution of metals under the influence of an electric current, followed by the formation of colloidal impurities. In the developed technology, it is proposed to use aluminum and duralumin waste.

The method provides high efficiency of water treatment and has significant advantages over reagent methods, primarily the compactness of the installation, the absence of bulky reagent facilities, and ease of maintenance, the ability to fully automate and save money.

Due to the inability to regularly provide the necessary reagent - aluminum sulfuric acid, we conducted research on obtaining the necessary reagent by electro coagulation using aluminum scrap as soluble electrodes.

\section{Experimental apparatus and procedure}

Was originally created and commissioned a pilot plant for producing coagulant to Tastes, a block diagram of the installation shown in Fig. 1.

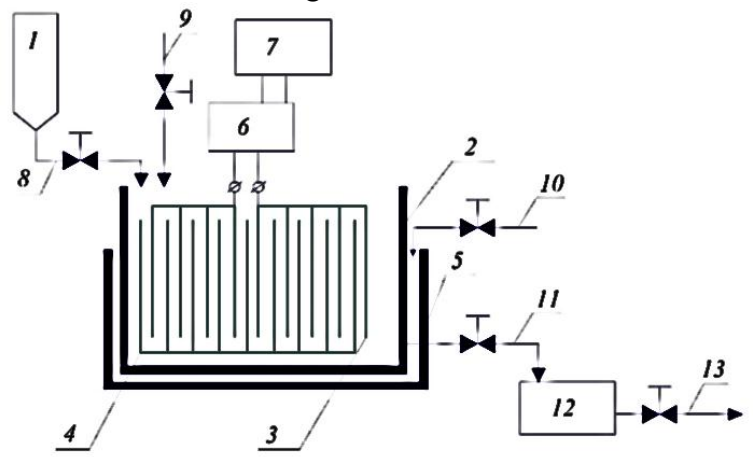

Fig. 1. Block diagram of the experimental unit for obtaining coagulant at TashTPP. 1-dimensional acid tank; 2-coagulator tank; 3-aluminum plates; 4-connecting tires; 5-cooling tank; 6-ballast resistance; 7-power supply; 8acid supply pipeline; 9 - water supply pipeline; $10-$ cooling water supply pipeline; 11 - ready coagulant discharge pipeline; 12 - ready coagulant container; 13coagulant supply to the technical water treatment line.

Based on the results of research at this plant, we proposed and agreed with TashTPP a draft project: "Creation of a highly efficient automated electro coagulation technology for pre-treatment and clarification of water for supply to steam boilers at TashTPP" [4, 5].

The block diagram of the proposed electro coagulation unit for pre-treatment and clarification of water is shown in Fig. 2. 
The process of pre-treatment and clarification of water is as follows. In one of the electro coagulator tanks (second tank is in reserve) is the time between coagulant. It should be noted that the activity of the reagent-derived coagulant. The mass consumption of substances used for coagulation is reduced by 2.5-5.0 times compared to reagent coagulation.

According to calculations, the tanks of electro coagulators should be chosen with a capacity of 2 cubic meters, the dissolved electrodes can be used in the form of pressed plates or sheets prepared from waste, or block-bulk. The tanks with electrodes receive an electrolyte from a tank in which the necessary density of the electrolyte is preprepared.

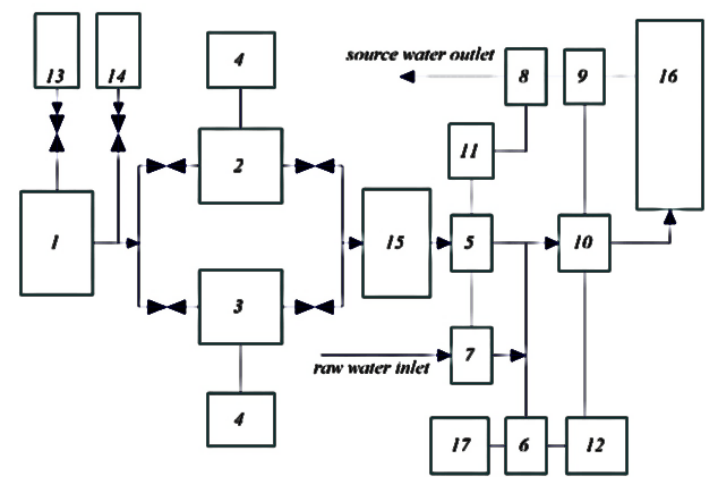

Fig. 2. Block diagram of the electro coagulation unit. The flowchart consists of the following equipment: 1 electrolyte preparation tank; 2 and 3 - electro coagulants; 4 - power supply; 5 - coagulant dispenser; 6 - acid dispenser; 7 - source water light sensor; 8 - water light sensor; 9 - clarified water sensor; 10 - source water sensor; 11 and 12 - dosing control circuits; 13 dimensional acid tank; 14 - finished salt tank; 15 coagulant accumulation tank; 16 - column-clarifier; 17 sulfuric acid tank.

\section{Results and analysis}

Power consumption can be minimized by selecting the interelectrode intervals and switching time of the electrodes. Calculations, based on the experience in a pilot plant for obtaining coagulant electrochemical method showed that the plant capacity at current density on the electrodes $50 \mathrm{~A} / \mathrm{m}^{2}$ and the temperature of the electrolyte $20{ }^{\circ} \mathrm{C}$ is equal to $20 \mathrm{l} / \mathrm{ch}$. The capacity of the unit can be regulated by the current density, as well as the temperature of the electrolyte.

The accumulated coagulant with the necessary composition is accumulated in the coagulant accumulation tank (available at TashTPP). From this tank, the coagulant is mixed with the source water through a dispenser (water from the Boz-su) is fed to the inlet of the clarifier column.

To improve the cleaning and clarification process, acid is added to the purified water until the required amount is obtained $p H$. Sensors $p H$ installed at the inlet and outlet of the columns-clarifier, and the control scheme of the dispenser regulates the flow of the required amount of acid through the dispenser.

In the automation scheme, it is planned to introduce control from the quality sensors of the source and clarified water. This will allow you to regulate the flow of coagulant in highly polluted source water and monitor the quality of clarified water during the day.

\section{Conclusion}

The feasibility of the proposed project is confirmed by a prototype of an electric coagulator, which has shown its efficiency, and it has been obtained a coagulant that fully meets the requirements of purification and clarification of the source water. Calculations, based on the experience in a pilot plant for obtaining coagulant electrochemical method showed that the performance of the proposed installation at current density on the electrodes $50 \mathrm{~A} / \mathrm{m}^{2}$ and the temperature of the electrolyte $20{ }^{\circ} \mathrm{C}$ is equal to $20 \mathrm{l} / \mathrm{ch}$ coagulant with chemical composition: density $1,3 \mathrm{gr} / \mathrm{sm}^{3}$; aluminum oxide and $\mathrm{Al}_{2} \mathrm{O}_{3} 7.6 \%$; in terms of aluminum sulphate $-\mathrm{Al}_{2}\left(\mathrm{SO}_{4}\right)_{3} 25.5 \%$. The capacity of the unit can be regulated by the current density, as well as the temperature of the electrolyte.

\section{References}

1. Kopylov, A. S., Lavygin, V. M., and Points, V. F., water Treatment in power engineering, Moscow: publishing house of MEI, 2006, 309 p.

2. Guzhelev E. P., Shalay V. V., Gritsenko V. I., Taran M. A. water Treatment and water-chemical modes in heat power engineering. Omsk: Omstu, 2005, 384 p.

3. Karpychev E. A. Investigation of coagulation of natural waters with an increased content of iron-organic compounds / autoref. Diss. Cand. tech. sciences'. 2013. $20 \mathrm{p}$.

4. Usmonov N.O., Normuminov J.A., Axmatova S.R. Multichannel measuring the water transparency to automate water clarification process water treatment plant TashTPP. «European Conference on Innovations in Technical and Natural Sciences». Proceedings of the 13th International scientific conference. Vienna, - 2017 - 117$180 \mathrm{pp}$.

5. Usmonov N.O., Normuminov J.A., Taniyev M.X. The sketch scheme of automatic system of monitoring and control the work of lighters in water preparing plant at the Tashkent thermal power station. «The Twelfth International Conference on Eurasian scientific development». «East West» Association for Advanced Studies and Higher Education GmbH, Vienna, - 2017 81-83 pp. 